UCRL-ID-131239

\title{
Engineering Analysis and Literature Review of the use of CORBA on Distributed Object-Oriented Systems
}

\author{
Fred Holloway
}

June 11, 1997

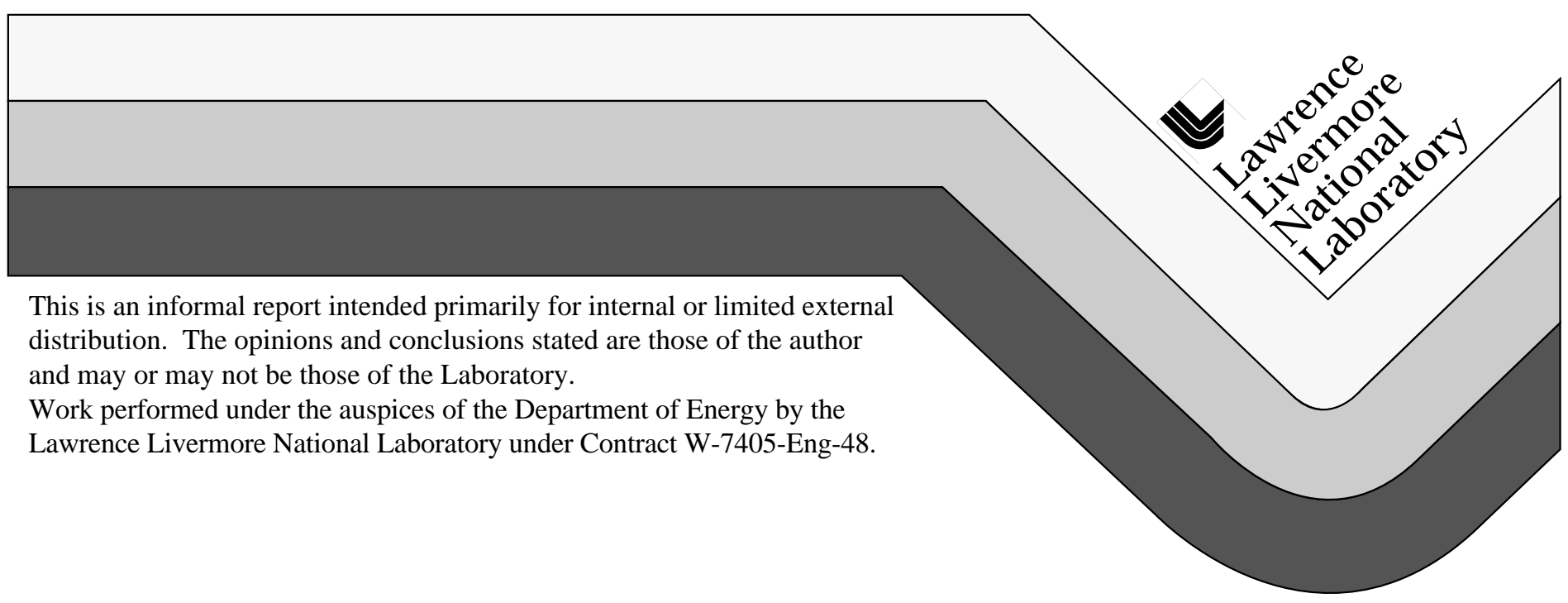




\section{DISCLAIMER}

This document was prepared as an account of work sponsored by an agency of the United States Government. Neither the United States Government nor the University of California nor any of their employees, makes any warranty, express or implied, or assumes any legal liability or responsibility for the accuracy, completeness, or usefulness of any information, apparatus, product, or process disclosed, or represents that its use would not infringe privately owned rights. Reference herein to any specific commercial product, process, or service by trade name, trademark, manufacturer, or otherwise, does not necessarily constitute or imply its endorsement, recommendation, or favoring by the United States Government or the University of California. The views and opinions of authors expressed herein do not necessarily state or reflect those of the United States Government or the University of California, and shall not be used for advertising or product endorsement purposes.

This report has been reproduced directly from the best available copy.

Available to DOE and DOE contractors from the Office of Scientific and Technical Information

P.O. Box 62, Oak Ridge, TN 37831

Prices available from (615) 576-8401, FTS 626-8401

Available to the public from the

National Technical Information Service

U.S. Department of Commerce

5285 Port Royal Rd.

Springfield, VA 22161 


\title{
Engineering Analysis and Literature Review of the use of CORBA in Distributed Object-Oriented Systems
}

\author{
NIF-0000314
}

29-April-1996

Revised 11/5/96

Revised 6/11/97

WBS 1.5

Fred Holloway

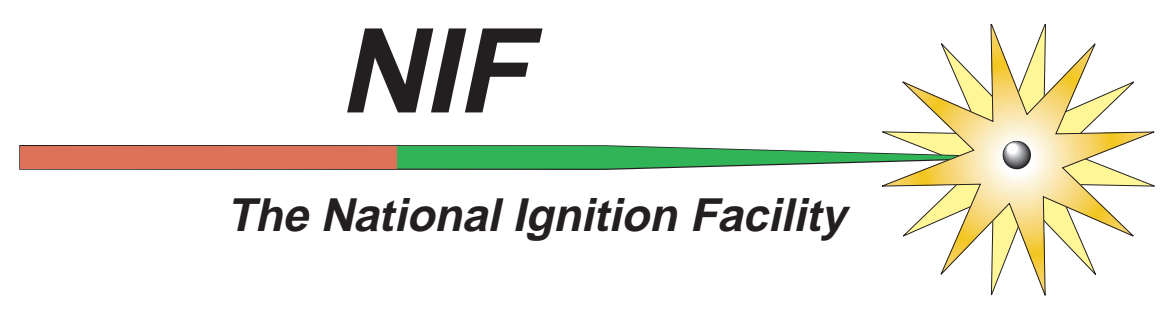


Table of Contents

1. Introduction 3

2. History and New Directions 3

2.1 Competing Paradigms 6

3. Requirements of a Component $\quad 7$

3.1 Opposing Methods of meeting requirements 9

4. Object Management Group (OMG) and Implementations 9

5. Overview of CORBA product Organization and General Services 9

5.1 Object Request Broker 9

5.2 Object Services $\quad 10$

5.3 Common Facilities $r$

6. Important 'Near' Parts of CORBA 12

6.1 Interface Definition Language (IDL) 13

6.2 Client Side Interface $r$

6.2.1 Client IDL Stubs 14

6.2.2 Dynamic Invocation Interface (DII) 14

6.2.3 Interface Repository Application Programming Interface 14

6.2.4 ORB Interface 15

6.3 Object Adapter 15

6.4 Server Side Interface $\quad 15$

6.4.1 Server IDL Stubs or Skeletons $\quad 15$

6.4.2 Object Adapter 15

6.4.3 Implementation Repository 16

6.4.4 ORB Interface 16

6.5 Dynamic Interfaces $\quad 16$

6.5.1 Dynamic Skeleton Interface (DSI) 16

6.6 Interface Repository 16

6.6.1 Interface Repository Navigation 18

7. Initialization and Connections 18

7.1 Component Initialization, Bootstrapping $r$

7.2 Interconnections of ORB's 19

8. The Total CORBA 19

9. Performance of CORBA 21

9.1 Our Measurements 21

10. Reliable Systems with CORBA 22

11. RT Operating System interface with CORBA 22

12. Suppliers and Users of CORBA 22

13. OpenDoc 23

14. References 24 


\section{Introduction}

This note was written based upon review of many papers, articles, and text books at time when we had little experience with an actual CORBA product. Some of the references conflicted with each other, and we now see that some of the comments made by other authors were misunderstandings or wrong. The product that we are currently using (and many others) do not implement all of CORBA - in fact, it is obvious that there is a great deal more to add to CORBA to meet all of the goals and expectations summarized herein.

With all of its capability and promised advantage, CORBA is a complex package of technologies and products which requires quite a concentrated effort to master.

We have developed a Test Package which to date has been used to send and recieve data from up to 12 Servers on up to 5 computers in our office network, with up to 1000 objects per server. The data is expressed in all available IDL types including long arrays and unbounded strings.

Performance measurements have established solid data upon which we can base estimates and models of the performance of the communication layer of the overall system after further design and prototyping on the frameworks and applications has been completed (see "CORBA Test Package, ref. 14).

\section{History and New Directions}

"Unless you spent the last year hiding on some deserted island, you probably know that there's a full-fledged object war going on. One side is Microsoft, with its COM/OLE component infrastructure and object bus - its the de facto object standard. On the other side is the rest of the industry with its support of the OMG's CORBA as the object bus - its the de jure object standard". (Ref. 1) Many people think that CORBA is much better than COM/OLE, at least technically. "CORBA is the most likely candidate for the improved infrastructure required to implement large-scale, mission-critical, object-oriented, portable applications that will span palmtop to supercomputers over the next 5 to 10 years" (ref. 11).

Terminology

$\mathrm{COM}=$ Component Object Model -- caution: Digital is developing with Microsoft a "Common Object Model (COM) which may be combined with Microsoft's COM.

OLE = originally "Object Linking and Embedding" (but it's not objectoriented) 
Microsoft recently announced "ActiveX", a slimmer, lighter, and renamed version of OLE redesigned to work over the World Wide Web (ref. 11). Microsoft is also shipping beta versions of Distributed Common Object Model (DCOM).

More recently, it appears that ActiveX is being overshadowed by Java - a sign of how fast the field is evolving.

CORBA $=$ Common Object Request Broker.

The current Ethernet era of client/server - which began about 10 years ago is coming to an end. Its being replaced by webs influenced by the exponential increase of low-cost bandwidth and a new generation of network-enabled multithreaded desktop operating systems with plug-andplay architecture. Millions of machines on the global 'information highway' can be both clients and servers.

The distributed object bus provides the essential middleware that will propel the dominance of distributed objects or components. Components are independently packaged objects of intelligence that can operate across languages, operating systems, networks, and tools. The object bus will support nested transactions that can span multiple servers, long-lived transactions that execute over long periods of time as they travel from server to server, queued transactions that can be used in secure business-tobusiness transactions, roaming agents that look over business interests, active multimedia compound documents that can be moved, stored, viewed, and edited-in-place anywhere on the network, and sniffer agents which sit on the network at all times collecting information for system management, looking at trends, gathering statistics.

The design of CORBA was derived from the inputs of many people representing the various technologies, developments, and competing paradigms of the past. The following figure illustrates some of the history leading CORBA. 


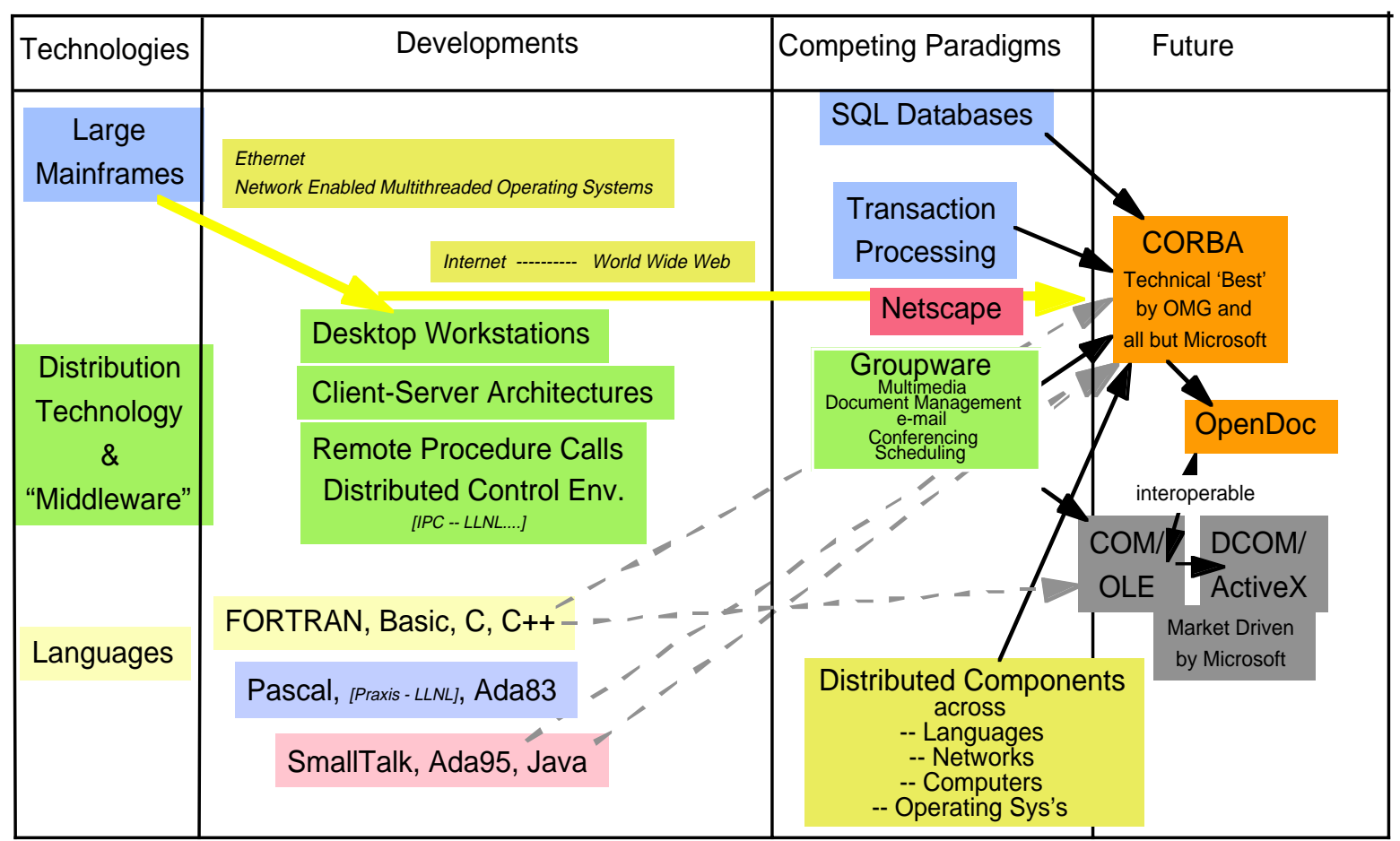

Figure: Historical Developments which led to CORBA 


\subsection{Competing Paradigms}

There are four competing paradigms for developing new client/server applications:

- SQL databases - by encapsulating SQL commands in named and compiled procedures that reside on the same server as the database (referred to as TP lite or stored procedures). SQL databases servers are the dominate model for creating client/server applications today. SQL is poor at managing processes - hence the event of SQL frontend tools like Power Builder. Also, different vendors SQL based products do not interoperate well.

- TP (Transaction Processing) Monitors

Transaction Processing Monitors have been used for many years on mainframes to manage processes and orchestrate programs by breaking complex applications into pieces called transactions and to many large applications to act in unison to service thousands of clients. They are injected between the remote clients and the server resources in a middle tier, provide routing, load balancing, funneling (dealing with a subset of 1000's of clients at any particular time), restart. The can manage transactional resources across multiple servers and can cooperate with other TP Monitors in federated arrangements. The architecture is similar in many ways to that needed for the distributed object era.

TP Monitor vendors were slow to adapt to the Ethernet era and shrink wrap market realities, and their products were overkill for the single-server/single-vendor, departmental sized applications that dominated the Ethernet era. TP Monitor vendors have a solid technical background to dominate in the distributed object era, and have been heavily involved in creating the CORBA standards. The next generation of TP Monitors will be called Object Request Brokers and will be packaged for the mass market.

- Groupware

Groupware is a collection of technologies that allows representation of complex processes that center around collaborative human activities. It includes:

- multimedia document management

- workflow (automatic routing of events of work)

- e-mail

- conferencing 
- scheduling

Groupware collects highly unstructured data - text, images, faxes, mail, and bulletin boards - and organizes it into a 'document' which can then be viewed, stored, replicated and routed anywhere on the network. The premier groupware product in industry is Lotus Notes.

- Distributed Objects or Components

Components are objects designed and implemented by any of many methodologies, combined with other objects that represent distribution technology to become standalone distributed objects that provide the unit of work and distribution in a plug-and-play manner across networks, applications, languages, tools, and operating systems.

\section{Requirements of a Component}

"Components are Objects (in the object-oriented design sense) with provisions and enhancements for distribution over many kinds of computers without concern for languages, operating systems, windowing systems, networks, tools, or hardware platforms". (ref. 12)

In principal the important requirements of a Component are listed in the following table. Note that these are goals and that no present implementation of CORBA provides the total set.

- Self-contained - It is a self-contained, shrink-wrappable, marketable entity

- Clean Interface - It has a well specified interface providing all necessary information for client use

- Limited Tasks - It performs a limited set of tasks

- Unanticipated combinations - It can be combined in unanticipated ways with other Components to form a complete application.

- Extendible - can be extended by inheritance from other Components and by polymorphism.

- Security - protects itself and its resources, authenticates itself to its clients and vice versa. Keeps audit trails of its use.

- Licensing - enforces licensing policies including per-usage metering. 
- Versioning - provides some form of version control and insure that its clients are using the expected version.

- Life cycle management - must manage its creation, destruction, and archival; be able to clone itself, externalize its contents, and move from one location to another.

- Support for Open tool palettes - a component is imported within a standard tool palette to be assembled with other components using drag-and-drop and other visual assembly techniques

- Event Notification - be able to notify interest Components when something of interest occurs.

- Configuration and property management - provide an interface to configure it properties and scripts.

- Scripting - a component permits itself to be controlled via scripting languages by being self-describing and supporting latebinding.

- Metadata and introspection - provide information about itself upon request including a description of its interface, attributes, and suites (methods?) it supports.

- Transaction control and locking - trasactionally protect its resources and cooperate with other Components to provide all or nothing integrity. Provide locks to serialize access to shared resources.

- Persistence - by able to save its state and later restore it.

- Relationships - be able to form dynamic or permanent associations with other Components and control other Components.

- Self Testing - provide and run its own diagnostics.

- Semantic messaging - be able to interact with the vocabulary of the particular suites and domain specific extensions it supports. (this is anti-intuitive to the purpose of Components - FH)

- Self Installing - be able to install itself and automatically register its factory with the Component Registry.

“Objects excite programmers (and geeks) who write software systems and applications for a living. Components excite users who have projects to finish as soon as yesterday, but who don't care about programming languages or protocols - they just want the components to be functional, fast, easy to use, seamless, and self-contained" (ref. 12). 


\subsection{Opposing Methods of meeting requirements}

There are two opposing methods of providing the distributed object bus and support that Components need to meet the above requirements.

One method is Microsoft's approach with the COM/OLE and ActiveX component infrastructure and object bus. The other is the OMG's CORBA. Most people believe that CORBA is much better than COM/OLE, at least technically.

\section{Object Management Group (OMG) and Implementations}

Since 1989, a consortium of object vendors - The Object Management Group (OMG) - have been developing specifications for an open software bus on which object components written by different vendors can interoperate across computers, networks, and operating systems. Some of the principal contributors to the specification were: Expersoft, IBM, IONA, DEC, HP, HyperDesk, NCR, Novell, Object Designs, Sun Microsystems and SunSoft. The resulting core concept is CORBA. The final CORBA 2.0 specification was released by the OMB in March 1996.

There are nearly a dozen CORBA implementations on the market including IBM/SOM, HP/ORB Plus, Digital/ObjectBroker, and Iona/Orbix. (we are currently using Iona/Orbix and Objective Interface Systems Orbix/Ada on the NIF Project). There are over 500 vendors working on CORBA-compliant software products. As in any product line there are various versions -- latest and best standard from OMG is CORBA 2.0, which these notes are based upon.

\section{Overview of CORBA product Organization and General Services}

Flexibility of overall system architecture, while maintaining standard conventions, is enhanced by using CORBA since the object implementations can be configured to run locally and/or remotely without affecting their implementation or use (ref. 8).

The presence of object-oriented methodology in CORBA is the result of necessity, not of choice. CORBA supports the important aspects of OO design (polymorphism, data encapsulation, and inheritance) between the boundaries of distributed objects.

\subsection{Object Request Broker}

The Object Request Broker (ORB) (also called an object buss) is the middleware that supports Component interoperations across machines, languages, operating systems, and networks. It makes different objects (and their associated data sets) reusable by different applications. The ORB 
intercepts calls of the Components, finds another Component that can implement the request, passes parameters, invokes its methods, and returns the result. Data encapsulation occurs because each client object knows little about the data it accesses when making requests of the respective objects through the ORB. Components exchange Metadata and discover each other by using services of the ORB. If one description of an object is designed to interface with the ORB, any object derived from that parent object will preserve its parents interface. Note that the ORB itself is a self-describing Component who's description is contained within the Interface Repository (described below) and following the CORBA conventions.

A gateway infrastructure allows linkage of different ORBs. An integration path allows linkage between CORBA ORB's and other object oriented approaches such as Microsoft's COM with OLE.

\subsection{Object Services}

Object Services are provided by their own objects to support interactions between other objects. Note that not all of the important characteristics of Components (listed above) are supported yet in CORBA. In particular, there is currently no explicit support for real-time guarantees, recovery from partial failures, group communications, or causal ordering of events (see "Reliable Systems with CORBA" below). Currently an inconsistent set of available features are supported within the various implementations of CORBA. For example, although it receives great attention in the literature, the Dynamic Invocation Interface is not yet supported in the OIS/Ada_95 product.

The object services include:

- Life Cycle - operations for creation, copying, moving, and deleting objects or groups of objects where groups are defined by the Relationship Service including containment and referential relationships. "Deep" operations are optionally allowed where all related objects are involved.

To create a new object, a client must find a factory object (meaning an object that is capable of instantiating an object of the desired type, allocating resources, obtaining an object reference, and registering the new object with the Object Adapter and Implementation Repository.

- Persistence - a single interface for storing components persistently.

- Naming - allows components to locate other components on the bus by name. Each named Component is a structure with an identifier string and a descriptive string such as a type definition. More than one name can be optionally associated with an object reference. Naming hierarchies can be created and clients can 
navigate through naming context trees in search of needed objects. To bind a name is to create a name-to-object association for a particular context. Names can be registered with the Properties Service allowing searches on properties such as time_last_modified, etc.

- Event Notification - (caution: this section needs updating by version 2.0 specification) allows components to dynamically register or unregister interest in specific events. Provides asynchronous interactions between anonymous objects (i.e. notification when things happen) using standard CORBA requests. Interactions are between suppliers and consumers through an event channel and can be either by pushing or pulling (polling). At present the service is minimal in that it doesn't support features commonly found in Message-Oriented Middleware (MOM) such as priorities, filtering, transaction protection, reception confirmation, time-to-live stamps, or queue management. (some of this may be added in the future).

- Concurrency Control - provides lock manager for transactions or threads (may be all that is needed for 'reservations'?)

- Transaction - provides two-phase commit coordination among recoverable objects using flat or nested transactions

- Relationship - creates dynamic associations (a.k.a. hyperlinks) between objects. Provides mechanisms for transversing the links that group objects. Can be used to enforce referential integrity constraints, track containment relationships, and other actions with any type of links between objects.

- Externalization - provides standard stream-like mechanism to get data in and out of an object. Mechanism is implemented in two steps: copy to stream, copy form stream to receiving object. And vice versa.

- Query - a superset of SQL for objects based upon the Object Query Language. Returns a collection of objects that satisfy the criteria specified via a select operation. Three interfaces provide operations on the result of a query. (1) CollectionFactory creates a new instance of an empty collection, (2) Collection defines operations to add, replace, retrieve, and remove members of a collection and insert at a particular location and create a movable pointer to navigate through the collection, and (3) Iterator provides operations to traverse a collection with Reset_to_start, Next_element, and a test for More_actions.

The Query Service also provides a Framework (see below) consisting of 5 interfaces for dealing with the preparation and execution of a query. 
- Licensing - meters the use of objects for charging to insure fair compensation. Provides a model for usage control of objects. Metering is per session, per node, per instance creation, and per site.

- Properties - dynamically associates named values or properties to any object.

- Trader (soon to be defined) - advertises object services and assists in finding them.

- Collections

- Security

- Time

- Change Management

Application objects are independently developed then CORBA services are mixed in by subclassing the original class and inheriting the services needed to make a Component. This is accomplished via IDL.

\subsection{Common Facilities}

Common Facilities are collections of IDL-defined Components that provide services of direct use to application objects. There are two categories:

Horizontal Facilities

- User Interface Services - in-place editing services similar to those provided by OpenDoc and OLE

- Information Management Services - compound document storage and data interchange facilities similar to those provided by OLE and OpenDOC

- System Management Services - define interfaces for managing, instrumenting, configuring, installing, operating, and repairing distributed object Components.

- Task Management Services - workflow, long transactions, agents, scripting rules, and e-mail.

Vertical - will provide IDL-defined interfaces for vertical market segments such as health, retail, finance, (and control systems.....)

\section{Important 'Near' Parts of CORBA}

The important parts of CORBA for our immediate purposes are shown in the following figure. "The best way to think of CORBA is as the universal "software bus". CORBA is a series of sophisticated, but standard sockets into which objects can "plug and play" to interoperate with one another. 
Even when made by different vendors, at different times, the object interfaces are standard enough to coexist and interoperate" (ref. 11). The interface types and methods between the Server Objects and the Client are defined using an industry standard Interface Definition Language (IDL). An IDL compiler provides all of the necessary interface code and templates into which use-specific statements are added.

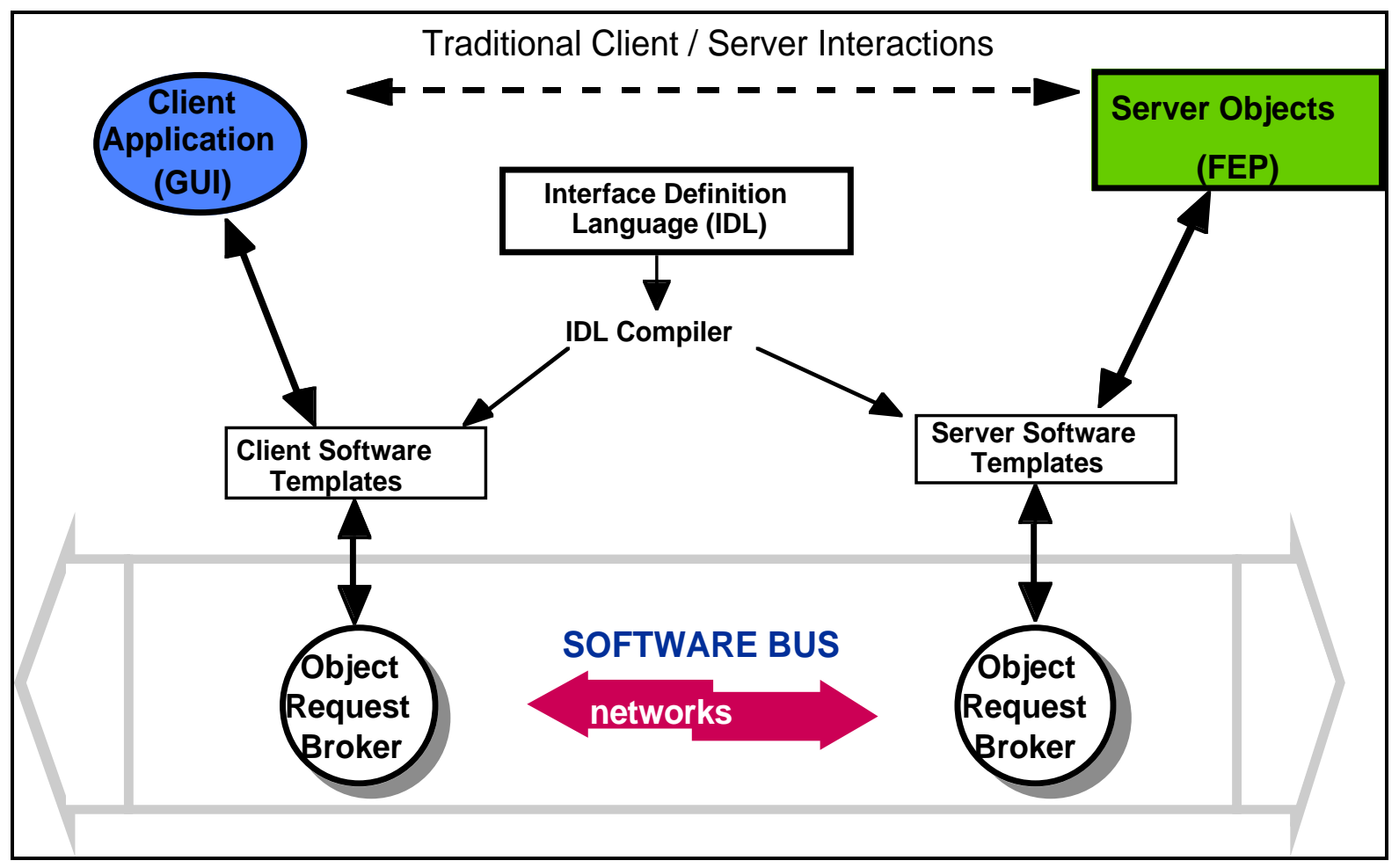

Figure : The important ports of CORBA for our Immediate Purposes

\subsection{Interface Definition Language (IDL)}

The Interface Definition Language (IDL) allows an object to interact with the rest of the world by communicating that object's methods and parameters to other objects through the ORB.

IDL statements specify a components attributes, the parent classes it inherits from, the exceptions it raises, the type events it emits, pragmas for generating globally unique identifiers, the methods its interface supports -including input and output parameters and their data types. IDL is a subset of $\mathrm{C}++$ with additional keywords to support distributed concepts; however, IDL does not include any procedural structures or variables. The goal of CORBA is to "IDL-ize" (sic) all client/server middleware and all components that live on an ORB.... It can be used to encapsulate legacy software. 
The metadata that describes the use of a component is generated automatically by an IDL compliant compiler or directly from an $\mathrm{OO}$ language or tool.

Pragma's (special instructions to a compiler) are used to set a prefix that is appended to all ID's, to set a version number, and to associate an arbitrary Repository ID with a specific IDL name.

\subsection{Client Side Interface}

\subsubsection{Client IDL Stubs}

The Client IDL Stubs provide a static interface to object services. The stubs act as a local call - a local proxy for a remote server object. A client has a stub for each interface it uses on the server. The stub includes code to perform marshaling (encoding/decoding the operation and its parameters into a flattened message that is sent/received to/from the server.)

\subsubsection{Dynamic Invocation Interface (DII)}

A Dynamic Invocation Interface (DII) is also provided which allows objects to dynamically define at run time the information which would have been provided statically by an IDL. DII allows an application to issue requests on objects whose interface may not have been defined at the time the application was compiled (ref. 13)

Explanation: the 'Common' in CORBA stands for the combination of two original submittals to the OMG on its Request for Proposal - a static approach by Sun and HP and a dynamic approach by Digital and HyperDesk.

Unlike IDL stubs which only allow RPC-style requests, the DII also allows clients to make non-blocking deferred synchronous (separate send and receive operations) and one-way (send-only) calls. (ref. $8<----$ this should be checked --- FH)

We may need to use the Dynamic Invocation Interface (DII)

- To avoid having to reboot the entire system when an interface definition changes. Perhaps FEP's can use a separate process for each component, then only reload the related process? But what about the rest of the system?

- How to handle exceptions (h/w error conditions) raised outside of the scope of an IDL stub (which only supports RPC-style requests which block the client until control is returned to the client) without DII?

\subsubsection{Interface Repository Application Programming Interface}

Provides services to obtain and modify the descriptions (metadata) of all registered Component interfaces, the methods they support, and the method signatures (parameters they require, etc.). 


\subsubsection{ORB Interface}

A direct interface to the ORB provides a few local services such as conversions from object reference to string and some language and system bindings handle differences of specific implementations.

\subsection{Object Adapter}

The Object Adapter is how a CORBA object knows of and use the services of an ORB.

\subsection{Server Side Interface}

\subsubsection{Server IDL Stubs or Skeletons}

Provide static IDL interfaces (Skeletons) to each service exported by the server.

\subsubsection{Object Adapter}

The Object Adapter contains the primary mechanisms for an object implementation to access ORB services and provides the environment for running the server application. The Object Adapter sits on top of the ORB's core communication services and accepts requests for service on behalf of the servers objects. Actions include:

- Registers server object implementation classes with the Implementation Repository

- Broadcasts the services it provides on the ORB, and responds to directory type queries

- Instantiates and activates new server objects at run time. The number of instances created is balanced as a function of the incoming client traffic load.

- Authenticates Client making the call. Security actions are left to the specific implementation.

- Generates and manages object references. Assigns references (unique ID's) to new objects and maps between implementationspecific and ORB-specific representations of object references.

- Processes incoming client calls. Peels off requests, and transfer it to the interface stub which interprets the request and incoming parameters and presents them to the objects method invocation.

The CORBA specified standard Object Adapter is called the Basic Object Adapter.

CORBA defines four object activation policies:

1. Shared server - multiple objects reside in the same server process (program) 
2. Unshared server - each object resides in a different server process (program)

3. Server-per-method - a new server is started for each request

4. Persistent server - servers are activated by means outside of the Basic Object Adapter

\subsubsection{Implementation Repository}

A run-time repository of information about the classes a server supports, the objects that are instantiated, and the ID's. Serves as a common place to store additional information associated with implementation, trace information, audit trails, security, and other administrative data.

\subsubsection{ORB Interface}

(Identical to that provided on the Client side)

\subsection{Dynamic Interfaces}

\subsubsection{Dynamic Skeleton Interface (DSI)}

The server side of the Dynamic Invocation Interface (DII). The DSI provides run-time binding and is used by the ORB to issue incoming method calls to objects that are implemented independently and do not have IDL-based compiled skeletons or compile-time knowledge of the implementations. They can be used by interpreters and scripting languages to dynamically generate object implementations.

But note that static interfaces are easier to program, provide more robust type checking, provide better performance, and are self-documenting in that one can tell what occurs by directly reading the code.

\subsection{Interface Repository}

Interface Repository - is a run-time database that contains dynamic metadata, machine readable (compiled) versions of the IDL's for the objects known by the ORB. These definitions may be captured directly from an IDL-compiler or through the Interface Repository write functions. Interface Repositories can be maintained locally or managed elsewhere. An ORB may access multiple Interface Repositories. An interface (or entry in the Interface Repository?) is defined for each of the 8 IDL structures:

1. Moduledef - defines a logical grouping of interfaces as a module

2. InterfaceDef - defines the objects interface, contains lists of constants, typedefs, exceptions, and interface definitions

3. OperationDef - defines a method at an objects interface, contains lists of parameters and exceptions raised.

4. ParameterDef - defines an argument of a method 
5. AttributeDef - defines the attributes of an interface

6. ConstantDef - defines a named constant

7. ExceptionDef- defines an exception that can be raised by an operation

8. TypeDef - defines the named types that are part of an IDL definition

Each Interface Repository is represented by global root object which contains the 8 possible IDL structures in the following hierarchy:

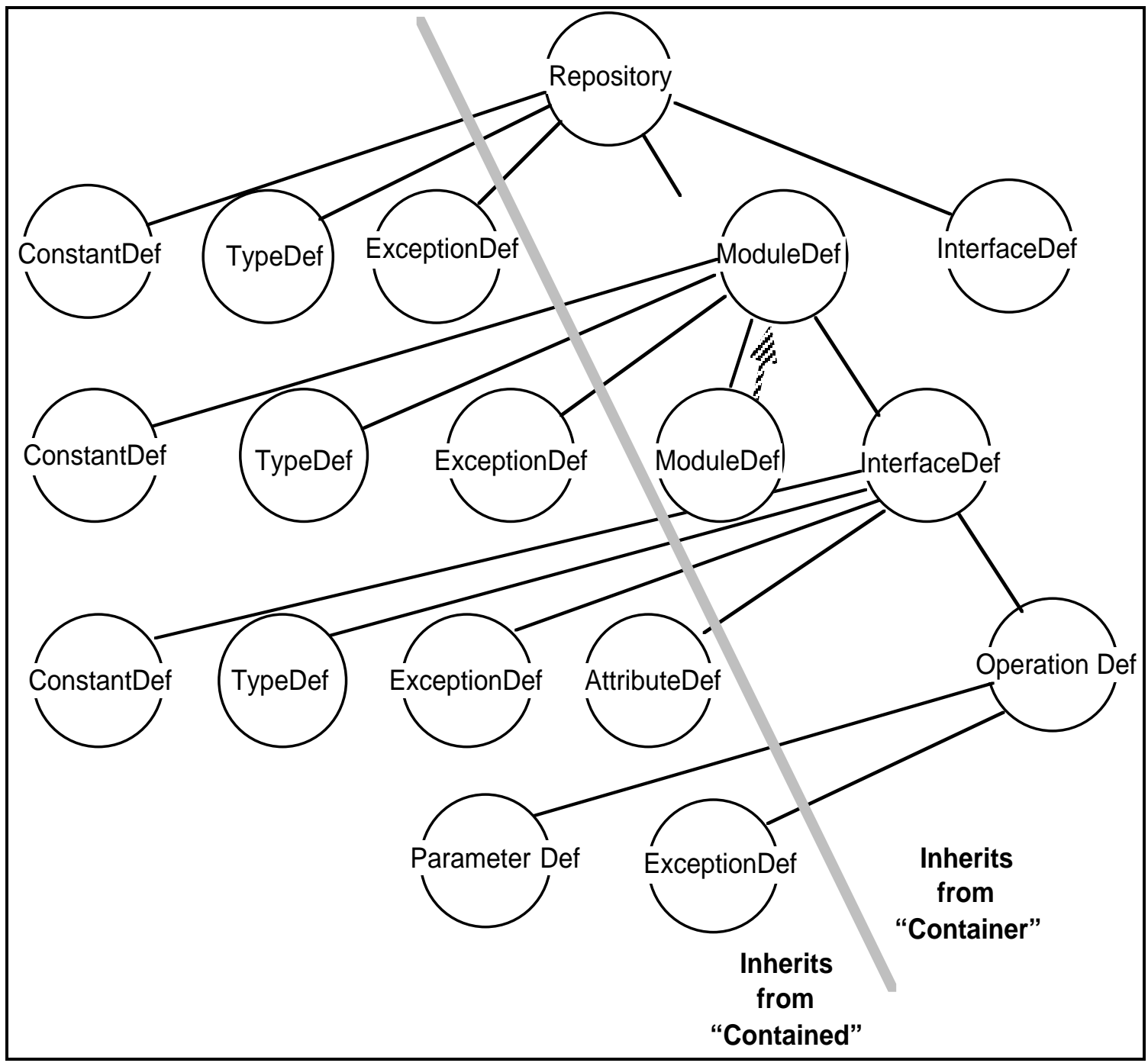

Figure: Containment \& Inheritance Hierarchy for the Interface Repository Classes 


\subsubsection{Interface Repository Navigation}

Navigation and extraction of information from the Interface Repository is accomplished with nine methods:

1. Describe - returns a Description structure containing the IDL that describes a contained object

2. Lookup - returns a sequence of pointers to objects within a contained object.

3. Lookup_name - locate a named object within a contained object.

4. Contents - returns a list of objects contained or inherited by the contained object

5. Describe_contents - returns a sequence of pointers to the content descriptions of objects within the contained object (a combination of Describe and Contents)

6. Describe_interface - returns a structure describing an InterfaceDef object

7. Is_a - returns TRUE if contained InterfaceDef is identical to or inherits directly from an interface specified in an input parameter

8. Lookup_ID - lookup an object in a Repository

9. Get_primative - obtain a reference to a primitive object of a Repository such as base data types and codes.

\section{Initialization and Connections}

\subsection{Component Initialization, Bootstrapping}

A Component in a CORBA compliant system performs the following initialization steps to bootstrap itself into operation:

- Informs ORB of presence via a CORBA API call to ORB_Init and obtain a reference to the ORB Component.

- Inform BOA of presence via invoking method BOA_Init within the ORB and to obtain its object reference.

- Invoke method List_Initial_Services to obtain a list of well-known objects, for example the Interface Repository and Naming Services.

- Invoke method Resolve_Initial_References to obtain references for the services required. 


\subsection{Interconnections of ORB's}

The General Inter-ORB Protocol (GIOP) specifies a set of seven message formats that cover all the ORB request/reply semantics to provide ORB-toORB interconnections over any transport protocol. A Common Data Representation (CDR) maps data types defined in the OMG IDL into a flat message representation.

CORBA 2.0 specified the mandatory Internet Inter-ORB Protocol (IIOP) which defined how GIOP messages are exchanged using TCP/IP connections, making it possible to use the Internet itself as a backbone ORB.

For application specific networks, Environment-Specific Inter-ORB Protocols (ESIOPs) are specified. The first ESIOP uses DCE where IDL and CDR types are mapped directly into DCE's native Network Data Representation (NDR). The DCE ESIOP provides a robust environment for mission-critical ORBs with a rich set of features including efficient large data transfers, Kerberos security, cell and global directories, distributed time, and authentication. Some of the major features within DCE such as Remote Procedure Calls are not currently applicable to CORBA and hence, even though very robust, represent unneeded overhead. DCE is supported by IBM, Digital, Tandem, and HP who will support it on their ORBs. Microsoft is attempting to create COM directly on top of DCE for worldwide (but proprietary) use.

\section{The Total CORBA}

The following figure illustrates the entire CORBA architecture 


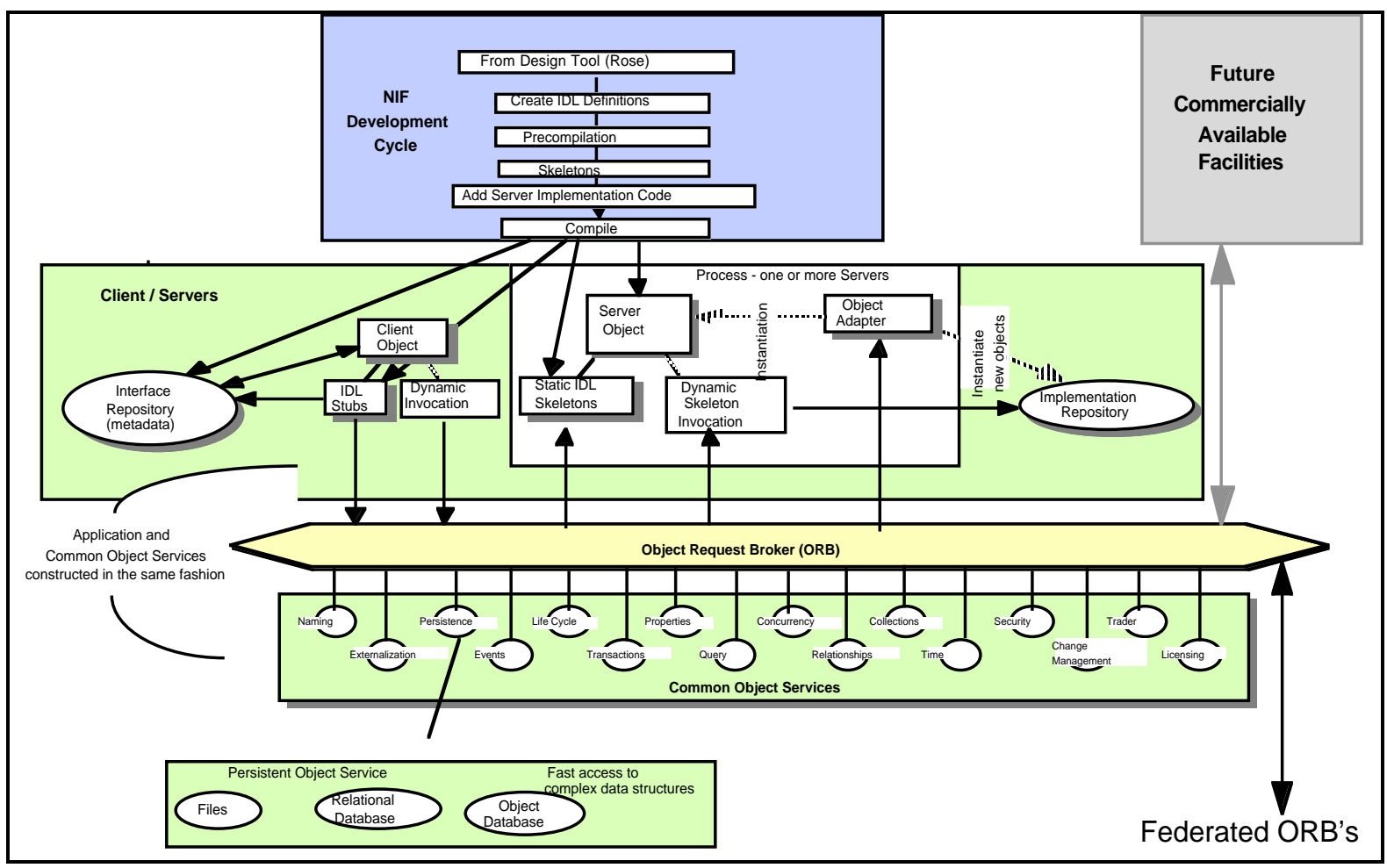

Figure: The entire CORBA architecture 


\section{Performance of CORBA}

The speed of performance of CORBA has been measured as about $1 / 3$ as fast as 'the best $\mathrm{C}++$ ' code for small data packets (1000 bytes) between two dualprocessor SPARCStation Model 712's connected with an ATM network (ref. 8). The top speed of simple non-structure data transfer of large blocks (100Kbytes) is about the same for $\mathrm{C}++$ code and CORBA (50 Mbytes per second). The reference contains many plots of performance of Orbix and ORBeline CORBA versus C++ versions of TTCP and RPC for buffer sizes from 1000 bytes to $140 \mathrm{Kbytes}$. Experience of the authors indicates that present CORBA implementations are well-suited for request/response applications over lower-speed networks (such as Ethernet) however considerable overhead is obvious within CORBA when higher speed networks are used. Overhead comes from a variety of sources: (1) non-optimized conversions, data copying, and memory management, (2) generation of non-word boundary aligned data structures by the CORBA stub compilers, (3) excessive control information carried in request messages, (4) inefficient and inflexible receiver-side demultiplexing and dispatching operations, (5) long chains of intra-ORB function calls, and (6) lack of integration with the underlying operating system mechanisms. (underlines are probably the areas that we need to be aware of -- FH)

"The latency for sending richly-typed data increases rapidly as the buffer size increases with Orbix" (ref. 10) Two-way richly-typed structures of 1000 bytes have a latency of about $45 \mathrm{~ms}$ on the above test platform. (we need to review this more carefully - FH)

Numerous suggestions are made in the referenced paper and others for optimizing CORBA including revisions of the specifications. (it appears that this work is ongoing and could well result in optimized versions of CORBA and better interfaces with the RT operating systems before we need it on NIF $-F H)$

\subsection{Our Measurements}

Subsequently to the above comments summarized from the literature, we have now measured the performance of the Objective Interfaces Systems (OIS) Ada_95 Object Request Broker software in combination with our own Test Package which performs I/O operations with 1000's of objects distributed over 5 computers using transaction record structures and information similar to those expected within the NIF control system. In summary, one can plan on roughly 1-2 ms of processor consumption at both the Server and Client ends plus the network transaction time. The Test Package and performance measurements are described in detail in "CORBA Test Package - First Milestone", F. Holloway, E. Stout, and M. Gorvad, NIF-0002349, June 6, 1997. (ref 14) 


\section{Reliable Systems with CORBA}

"Neither the CORBA standard nor conventional implementations of CORBA directly address complex problems related to distributed computing such as real-time, high-speed, quality of service, partial failures, group communications, and causal ordering of events (ref. 9). The CORBA model itself does not provide solutions to the problem of detecting and reacting to partial failures, and there is need for distributed debugging tools and run-time validation tools. The reference proposes extending the CORBA ORB (using OO extensions), to add appropriate lessons learned from other models) to increase the reliability and availability of CORBA based interactions and systems (again, it appears that this work is ongoing and could well result in improved versions of CORBA before we need it on NIF -- FH)

\section{RT Operating System interface with CORBA}

"The performance of current CORBA implementations is not suitable for latency-sensitive real-time applications, including real-time systems (e.g. avionics), and constrained latency systems (e.g., teleconferencing) (ref. 10). The interface between CORBA implementations and underlying operating systems is inefficient in many respects. Areas identified for improvement include: resource scheduling mechanisms, multiplexing, data copying, and byte-order alignment conversions (which often occur at several layers), and the lack of ability to select between using compiled code versus interpreted code for conversions.

\section{Suppliers and Users of CORBA}

"Vendors are shipping CORBA-compliant ORBs on all major operating systems from the Apple Macintosh OS, Windows (all flavors), more than 20 different UNIX operating systems, to Digitals OpenVMS and IBM's MVS" (ref. 11) (we have not seen much of this yet except for IONA -- FH)

Identified vendors and CORBA-compliant ORB products include:

- BBN's (Corbus)

- Component Integration Laboratories (OpenDoc)

- Chorus Systems (CHORUS/COOL ORB)

- Digital Equipment Corporation's (Object Broker)

- DNS Technologies (SmalltalkBroker)

- Expersoft (PowerBroker CORBAplus)

- Hewlet Packard (ORBplus)

- Iona (Orbix)

- IBM (Distributed System Object Model -DSOM)

- Object Interface Systems (Orbix/Ada) (being used on NIF) 
- Object-Oriented Technology (Distributed Object Management Environment - DOME)

- O/SPACE (CORBA implementation for Java)

- Sun Microsystem (NEO)

- Tandem (nonStop DOM)

- TRW (Universal Network Architecture Service - UNAS)

- Xerox PARC (ILU)

Recent announcements of important users, applications, and tools include:

- Chevron has announced one of the largest examples to date of plans to use CORBA technology to link engineers' desktop systems with browsers to geographical, seismic, and historical drilling information stored in databases on many kinds of computer platforms all over the world. They have selected the IONA CORBA implementation. (Computer World, April 28, 1997)

- The Gap has 1900 stores around the world and posted \$5.3 billion in sales in 1996. Keeping everyone connected to the most up-to-date information on products and sales is important. They use IBM mainframes at the backend, Sun Solaris servers at the middle tier, and OS/2 and Windows NT- based desktops. A corporate decision was made to go to object-oriented technology and to access information with browser technology by using CORBA with Java. They have selected the Visigenic's CORBA software. "Putting this OO system together is a big job - a lot of pieces and ways to go wrong" (Phil Wilkerson, The Gap Inc., Computer World, June 9, 1997) The switch over to the new system is expected in 6-8 months.

\section{OpenDoc}

Note: This was almost removed from recent version since in the past year OpenDoc appears to be almost totally replace by web browsers with Java capability.

"OpenDoc has been called "a CORBA object with desktop smarts" (ref. 12). OpenDoc is object-oriented and 'network-distributed' to its core. It was designed to operate over wide area networks on completely different types of computers. OpenDoc also has interoperability with Microsoft's OLE as a primary design goal. OLE objects can be embedded into OpenDoc documents. OpenDoc is important because it will be a primary way for CORBA to get to the desktop of non-Microsoft platforms (the other path is NetScape's support for the Internet InterOrb Protocol in future browers).

OpenDoc documents can contain any number or variety of text, spreadsheets, video and audio clips, graphics, CAD/CAM drawings -- even other 
compound documents. The fact that OpenDoc is designed to be independent of specific operating system is a critical difference with Microsoft's OLE/DCOM.

The OpenDoc standard was developed by the Component Integration Laboratory - a non-profit industry association started in 1993 by Apple, IBM, SunSoft, Oracle, Novell, WordPerfect, Xerox, and Taligent. An independent certification program now exists to assure developers of compatibility with the standard. Certified parts are called 'Live Objects'.

\section{References}

1. "The Essential Distributed Objects Survival Guide”, R. Orfali, et.al. Wiley, 1996.

2. Programming with CORBA, Gabriel Minton, Unix Review, April 1996

3. Notes on OLE and CORBA, D Katiyar, Datiyar@eng.sun.com

4. The Common Object Request Broker, v2.0, OMG

5. CORBA Services, 3/28/96 OMG

6. Common Facilities Architecture, 11/95 OMG

7. How do we Integrate a control system containing 200 computers and 1000 processes, Jim Spann, 10/12/95

8. "Measuring the Performance of Communications Middleware on High-Speed Networks, A Gokhale and D Schmidt, Dept. of Computer Science, Washington University, St. Louis, MO (http://www.cs.wustl.edu/ schmidt/)

9. Constructing Reliable Distributed Communications Systems with CORBA, S. Maffeis and D Schmidt. to be publilshed in IEEE Communications Magazine, Vol 14, No 2, February 1997.

10. Operating System Support for High-Performance, Real-Time CORBA, A Gokhole, D. schmidt, T. Harrison, G Parulkar, Proceedings of the 5th International Workshop on ObjectOrientation in Operating Systems, October 1996, Seattle, Washington.

11. "Collaborating Distributed Objects Over the Web", Rich Lysakowski, Scientific Computing \& Automation, August 1996.

12. "OpenDoc and the Move Towards Component-Based Documentcentric Software", Rich Lysakowski, Scientific Computing \& Automation, October 1996. 
13. "Programming Guide, orbix 2" IONA technologies Ltd. Oct 1996, pg 2.

14. "CORBA Test Package - First Milestone", F. Holloway, E. Stout, and M. Gorvad, NIF-0002349, June 6, 1997. 


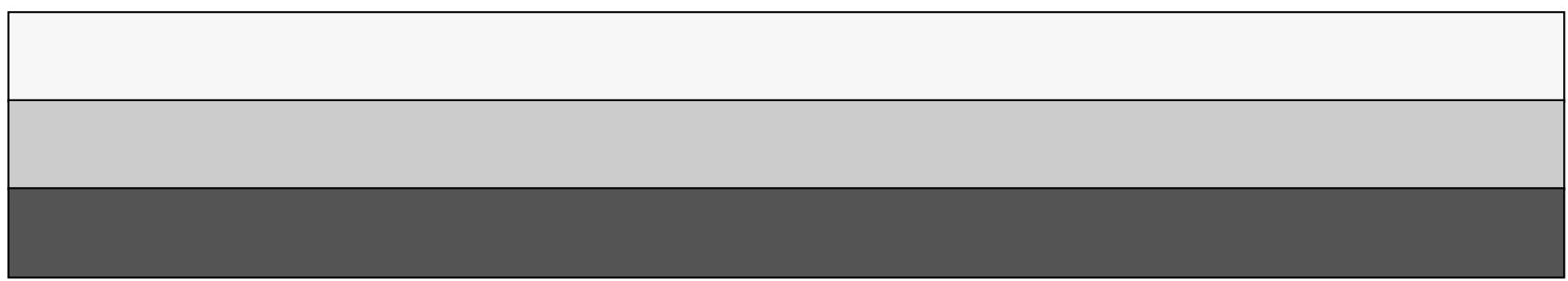

